\title{
Comparação da estrutura populacional das espécies de Uca (Crustacea: Decapoda: Ocypodidae) no Manguezal de Itacuruçá, Rio de Janeiro, Brasil
}

\author{
Luciane M. Bedê; Lídia M. Y. Oshiro; Luziane M. D. Mendes \& Alessandra A. Silva
}

Estação de Biologia Marinha, Universidade Federal Rural do Rio de Janeiro. Rua Sereder, 23860-000 Itacuruçá, Mangaratiba, Rio de Janeiro, Brasil. E-mail: lucianebede@ibest.com.br

\begin{abstract}
Comparison of the population structure of the species of Uca (Crustacea: Decapoda: Ocypodidae) in the mangrove of Itacuruçá, Rio Janeiro, Brazil. This study was conducted in the Itacuruçá mangrove, in the Sepetiba Bay, Rio de Janeiro, Brazil, with the objective of investigating the population structure of the species of Uca Leach, 1814. Sampling was carried out monthly from June, 2005 to May, 2006, during low tides. Crabs were captured manually by two people for a period of $15 \mathrm{~min}$ each. A total of 2580 crabs were obtained, of which 1465 were males and 1115 were females. The size of the fiddler crabs in the mangrove of Itacuruçá were the smallest reported so far in Brazilian mangroves. However, males attained a larger size than females. The size frequency distribution was unimodal for most of species, with the exception of $U$. thayeri Rathbun, 1900 and $U$. vocator (Herbst, 1804), which did not show a clearly-defined pattern. Males were most abundant among the largest size classes. The sex ratio differed significantly from 1:1 (male: female), most often with a higher frequency of males, except for $U$. thayeri and $U$. victoriana von Hagen, 1987, in which females were predominant.
\end{abstract}

KEY WORDS. Fiddler crab; frequency distribution; Sepetiba Bay; sex ratio; size.

RESUMO. Este trabalho foi realizado no Manguezal de Itacuruçá, na Baía de Sepetiba com o objetivo de analisar a estrutura populacional das espécies de Uca Leach, 1814. Foram realizadas coletas de junho/2005 a maio/2006, durante as marés baixas. Os caranguejos foram capturados manualmente por duas pessoas e durante 15 minutos. Um total de 2580 animais foi coletado, sendo 1465 machos e 1115 fêmeas. Com relação ao tamanho dos indivíduos, observou-se que os animais do Manguezal de Itacuruçá, de maneira geral, apresentam tamanhos menores que os encontrados em outros manguezais do Brasil. Contudo, os machos atingiram tamanhos maiores do que as fêmeas. A distribuição de freqüência em todas as classes de tamanho foi unimodal para a maioria das espécies, com exceção de $U$. thayeri Rathbun, 1900 e U. vocator (Herbst, 1804), as quais não apresentaram um padrão definido. Os machos foram mais abundantes em todas as classes de maiores tamanhos. A razão sexual diferiu significativamente da proporção 1:1, estando deslocada para uma maior freqüência de machos, com exceção de U. thayeri e U. victoriana von Hagen, 1987, as quais tiveram predominância de fêmeas.

PALAVRAS-CHAVE. Baía de Sepetiba; caranguejo-violinista; distribuição de freqüûncia; proporção sexual; tamanho.

No Brasil, dez espécies de Uca Leach, 1814 são encontradas ao longo da costa brasileira: $U$. burgersi Holthuis, 1967; $U$. cumulanta Crane, 1943; U. leptodactyla Rathun, 1898; U. maracoani (Latreille, 1802-1803); U. mordax (Smith, 1870); $U$. rapax (Smith, 1870); U. thayeri Rathbun, 1900; U. uruguayensis Nobili, 1901; U. victoriana von Hagen, 1987 e U. vocator (Herbst, 1804). Destas, somente $U$. victoriana não havia sido registrada até o momento no Estado do Rio de Janeiro, tendo sua distribuição limitada ao Estado do Espírito Santo (Melo 1996).

Os caranguejos $U c a$, conhecidos popularmente como chama-maré ou violinista, são caracterizados por um forte dimorfismo sexual, no qual os machos apresentam uma das quelas mais desenvolvida, compreendendo quase a metade da massa corporal do animal, enquanto as fêmeas apresentam os dois quelípodes do mesmo tamanho (Crane 1975).
Os chama-marés estão entre os habitantes mais familiares das áreas de estuários (Crane 1975, Frith \& Brunenmeister 1980), exercendo um importante papel estrutural e funcional na ecologia dos manguezais (Lee 1999, Botto \& Iribarne 2000, Meziane et al. 2002). Segundo Allen \& Curran (1974), os caranguejos violinistas, ao removerem a terra, promovem uma grande bioperturbação, a qual auxilia na ciclagem de nutrientes e de energia no ambiente (SKov et al. 2002, Asтноn et al. 2003).

A caracterização das populações naturais é um fator importante para compreender sua estabilidade ecológica. As variações sazonais da estrutura da população, densidade, razão sexual, recrutamento juvenil e intensidade reprodutiva, juntos com estimativas de migração, taxas de nascimento e mortalidade, são os aspectos mais freqüentes em estudos de biologia populacional (Jones \& Simons 1983, SANTOS et al. 1995). 
Com relação aos trabalhos realizados no Brasil, que abordam a biologia populacional do gênero $U c a$, podem ser citados aquele realizado por Castiglioni \& Negreiros-Fransozo (2006a) com $U$. rapax em um estuário degradado de Paraty no Rio de Janeiro e Costa \& Negreiros-Fransozo (2003) com U. thayeri, Colpo \& Negreiros-Fransozo (2004) com U. vocator, Costa et al. (2006) com $U$. thayeri e $U$. uruguayensis e Castiglioni \& NegreirosFransozo (2006b), todos realizados no litoral paulista.

Embora, no manguezal de Itacuruçá, sejam encontradas em elevada freqüência, quase todas as espécies de caranguejos de Uca do Brasil (Oshiro et al. 1998), existem poucos estudos sobre suas populações. Assim, este trabalho tem como objetivo comparar a estrutura populacional de oito espécies de Uca no manguezal de Itacuruçá, Rio de Janeiro, Brasil.

\section{MATERIAL E MÉTODOS}

A área selecionada para o presente estudo foi o manguezal de Itacuruçá $\left(43^{\circ} 05^{\prime} 30^{\prime \prime} \mathrm{W}, 22^{\circ} 55^{\prime} 00^{\prime \prime} \mathrm{S}\right)$, sendo as coletas realizadas mensalmente de junho/2005 a maio/2006, preferencialmente nas marés baixas. Os animais foram capturados manualmente e a unidade de esforço definida pela captura ao acaso de caranguejo durante 15 minutos e realizado por duas pessoas como sugerido por Costa \& Negreiros-Fransozo (2003). O tempo de esforço amostral foi definido com base em trabalhos anteriores realizados com o mesmo gênero neste manguezal por Oshiro et al. (1998) e BEDÊ et al. (2007).

Os caranguejos foram colocados em sacos plásticos, etiquetados e levados para laboratório na Estação de Biologia Marinha, UFRRJ), onde foram mantidos no freezer até o processamento.

No laboratório os animais foram triados, identificados, separados por sexo e a largura da carapaça (LC) foi medida com um paquímetro de precisão $0,1 \mathrm{~mm}$. Alguns animais, devido ao pequeno tamanho, foram medidos com o auxílio de uma ocular micrométrica de um microscópio estereoscópico. Os animais foram agrupados em 8 a 12 classes de tamanho, com intervalos de 0,5 $\mathrm{mm}, 1 \mathrm{~mm}$ e $2 \mathrm{~mm}$ dependendo da espécie em estudo.

Após o processamento, os caranguejos foram fixados em formol a $10 \%$ e posteriormente conservados em álcool a 70\%.

As espécies de Uca foram identificadas seguindo as chaves propostas por Melo (1996) e Crane (1975). Os caranguejos cuja identificação apresentou dúvida foram enviados para o Museu de Zoologia da Universidade de São Paulo, identificados pelo Dr. Gustavo A.S. de Melo e depositados sob o número MZUSP - 17.163.

O teste do Qui-quadrado foi utilizado para testar a razão sexual esperada de 1:1 e se, esta razão sexual diferiu em cada mês de estudo. O tamanho médio dos machos e fêmeas de cada espécie foi comparado usando o teste $t$ de Student (ZAR 1984).

\section{RESULTADOS}

Foram coletadas, oito espécies de Uca: U. cumulanta, $U$. leptodactyla, U. mordax, U. rapax, $U$. thayeri, $U$. uruguayensis, $U$. victoriana e $U$. vocator. Foram amostrados 2580 caranguejos, sendo 1465 machos e 1115 fêmeas. A espécie encontrada com maior abundância no Manguezal de Itacuruçá foi $U$. rapax, com 685 exemplares, sendo 414 machos e 271 fêmeas, seguida por U. leptodactyla (541), com 253 machos e 288 fêmeas, e com a menor abundância $U$. uruguayensis (81), com 57 machos e 24 fêmeas e $U$. vocator (36), com 30 machos e 6 fêmeas.

A análise da comparação do tamanho médio entre machos e fêmeas de cada espécie demonstrou que os machos eram significativamente maiores $(t, \mathrm{p}<0,05)$, nas populações de $U$. cumulanta, $U$. mordax e $U$. rapax. Já as fêmeas apresentaram maior tamanho médio $(t, \mathrm{p}<0,05)$ maior tamanho médio nas populações de $U$. thayeri e $U$. victoriana. Não se observou diferenças de tamanho entre sexos nas populações de $U$. leptodactyla, $U$. uruguayensis e $U$. vocator $(t, \mathrm{p}>0,05)$ (Tab. I).

Com exceção de $U$. leptodactyla, as demais espécies apresentaram diferenças significativas de razão sexual $\left(\chi^{2}, p<0,05\right)$. Contudo, esta proporção esteve desviada de maneira geral para os machos. As fêmeas foram significativamente predominantes, apenas para $U$. thayeri e $U$. victoriana. Essa diferença na proporção de machos e fêmeas também foi muito comum ao longo do ano amostral, embora diferença significativa não tenha sido observada em todos os meses (Tab. II).

Tabela I. Número de indivíduos (n), largura média da carapaça (LC médio), desvio padrão (DP), menor LC (< LC), maior LC (> LC), em machos e fêmeas das espécies de Uca, coletados no Manguezal de Itacuruçá, no período de junho de 2005 a maio de 2006.

\begin{tabular}{|c|c|c|c|c|c|c|c|c|c|c|}
\hline \multirow{2}{*}{ Espécies } & \multicolumn{5}{|c|}{ Machos } & \multicolumn{5}{|c|}{ Fêmeas } \\
\hline & $\mathrm{n}$ & LC médio & DP & $<\mathrm{LC}$ & $>\mathrm{LC}$ & $\mathrm{n}$ & LC médio & DP & $<\mathrm{LC}$ & $>\mathrm{LC}$ \\
\hline U. cumulanta & 306 & 4,58 & 0,88 & 2,75 & 7,57 & 79 & 3,86 & 0,88 & 2,08 & 6,86 \\
\hline U. leptodactyla & 253 & 8,74 & 1,68 & 3,43 & 14,29 & 288 & 8,40 & 1,55 & 3,14 & 12,57 \\
\hline U. mordax & 301 & 12,11 & 2,54 & 4,57 & 20,79 & 165 & 11,65 & 2,06 & 5,71 & 19,91 \\
\hline U. rapax & 414 & 10,67 & 2,95 & 4,00 & 19,50 & 271 & 9,29 & 2,72 & 2,58 & 16,76 \\
\hline U. thayeri & 74 & 9,35 & 5,82 & 4,31 & 25,91 & 124 & 11,26 & 6,04 & 2,58 & 25,78 \\
\hline U. uruguayensis & 57 & 7,14 & 1,42 & 4,57 & 12,00 & 27 & 7,83 & 1,52 & 5,00 & 10,86 \\
\hline U. victoriana & 30 & 4,58 & 0,92 & 2,67 & 6,71 & 158 & 5,33 & 1,38 & 2,83 & 10,14 \\
\hline U. vocator & 30 & 14,76 & 2,63 & 9,51 & 18,08 & 6 & 15,46 & 3,16 & 10,6 & 19,96 \\
\hline
\end{tabular}

Revista Brasileira de Zoologia 25 (4): 601-607, December, 2008 
Tabela II. Variação mensal da proporção sexual das espécies do gênero Uca coletadas de junho de 2005 a maio de 2006 no Manguezal de Itacuruçá.

\begin{tabular}{|c|c|c|c|c|c|c|c|c|}
\hline Meses & U. rapax & U. leptodactyla & U. mordax & U. cumulanta & U. thayeri & U. victoriana & U. uruguayensis & U. vocator \\
\hline Jun & $1: 1,6$ & $1: 2$ & $1: 0,83$ & $1: 0,43^{*}$ & $1: 2$ & $1: 18^{*}$ & $1: 0^{*}$ & $1: 0$ \\
\hline Jul & $1: 1,12$ & $1: 1,54$ & $1: 0,67$ & $1: 0,46^{*}$ & $1: 4$ & $1: 3^{*}$ & $1: 0,4$ & $1: 0$ \\
\hline Ago & $1: 1,29$ & $1: 0,13^{*}$ & $1: 1$ & $1: 0,07^{*}$ & $1: 0,6$ & $1: 0,92$ & $1: 0,75$ & $1: 0,3$ \\
\hline Set & $1: 0,76$ & $1: 1,8$ & $1: 0,41^{*}$ & $1: 0,83$ & $1: 3$ & $1: 4$ & $1: 0,6$ & $1: 1$ \\
\hline Out & $1: 0,67$ & $1: 0,67$ & $1: 0,23^{*}$ & $1: 0,5$ & $1: 3,5^{*}$ & $1: 1,67$ & $1: 0,5$ & $1: 0$ \\
\hline Nov & $1: 0,77$ & $1: 0,75$ & $1: 0,42^{*}$ & $1: 0,24^{*}$ & $1: 3,2^{*}$ & $1: 15$ & $1: 0$ & $1: 6^{*}$ \\
\hline Dez & $1: 1,05$ & $1: 1,56$ & $1: 0,48^{*}$ & $1: 0,23^{*}$ & $1: 1,67$ & $1: 22^{*}$ & $1: 0^{*}$ & $1: 1$ \\
\hline Jan & $1: 0,38^{*}$ & $1: 1,56$ & $1: 0,37^{*}$ & $1: 0,15^{*}$ & $1: 1,57$ & $1: 18^{*}$ & $1: 0,75$ & $1: 0^{*}$ \\
\hline Fev & $1: 0,37^{*}$ & $1: 0,8$ & $1: 1$ & $1: 0,33^{*}$ & $1: 1$ & $1: 3$ & $1: 1,2$ & $1: 0$ \\
\hline Mar & $1: 0,31^{*}$ & $1 ; 1,26$ & $1 ; 1$ & $1 ; 0,16^{*}$ & $1 ; 0,94$ & $1 ; 8,5^{*}$ & $1 ; 0$ & $1 ; 0,1^{*}$ \\
\hline Abr & $1: 0,52$ & $1: 0,45^{*}$ & $1: 0,57$ & $1: 0,09^{*}$ & $1: 1,13$ & $1: 12^{*}$ & $1: 1$ & $1: 0$ \\
\hline Mai & $1: 0,45$ & $1: 1,39$ & $1: 1,05$ & $1: 0,14^{*}$ & $1: 0$ & $1: 10^{*}$ & $1: 0$ & $1: 0$ \\
\hline Total & $1: 0,65^{*}$ & $1: 1,14$ & $1: 0,55^{*}$ & $1: 0,26^{*}$ & $1: 1,68^{*}$ & $1: 5,3^{*}$ & $1: 0,42^{*}$ & $1: 0,17^{*}$ \\
\hline
\end{tabular}

*Diferença significativa $\left(\chi^{2}, p<0,05\right)$.

A distribuição de freqüência dos indivíduos, em classes de tamanho, foi unimodal, tanto para machos quanto para fêmeas para todas as espécies, com exceção de $U$. thayeri e de $U$. vocator, que não apresentaram um padrão de distribuição definido (Fig. 1).

A espécie Uca leptodactyla foi a única que apresentou classe modal similar (9,0 a 10,0 $\mathrm{mm}$ de LC), tanto para os machos como para as fêmeas, enquanto todas as outras espécies com distribuição unimodal, as fêmeas sempre apresentaram como classe modal, uma classe abaixo àquela dos machos (Fig. 1).

A população de $U$. mordax apresentou tanto para machos como para fêmeas, o maior tamanho em relação à classe modal de 10,0 a 12,0 mm de largura da carapaça nas fêmeas e de 12,0 a 14,0 mm de LC nos machos. Entretanto, U. cumulanta apresentou o menor tamanho em relação à classe modal para os machos na classe de 4,0 a 4,5 $\mathrm{mm}$ de LC e para as fêmeas na classe de 3,5 a 4,0 mm de LC (Fig. 1).

A única espécie que apresentou diferença na distribuição entre os sexos nas classes de tamanho foi $U$. uruguayenis, sendo unimodal para os machos, como nas outras espécies, e bimodal para as fêmeas. A maior freqüência de machos ocorreu na classe de 7,0 $\dashv 8,0 \mathrm{~mm}$ de LC e para as fêmeas nas classes de $5,0 \dashv 6,0$ e 7,0 $\dashv$, $0 \mathrm{~mm}$ de LC. Convém lembrar que foram poucos os espécimes de $U$. uruguayensis, o que pode estar afetando a distribuição das fêmeas da população, já que a bimodalidade é pouco perceptível (Fig. 1).

\section{DISCUSSÃO}

Nas populações de $U$. rapax, $U$. leptodactyla, $U$. mordax e $U$. cumulanta os machos foram maiores do que as fêmeas, ao contrário de $U$. thayeri, $U$. victoriana, $U$. vocator e $U$. uruguayensis, que apresentaram fêmeas com tamanhos maiores. Uca lepto- dactyla, $U$. uruguayensis e $U$. vocator não apresentaram variação com relação ao tamanho de machos e fêmeas.

Muitos autores, ao estudarem populações de espécies de Uca também observaram a existência de dimorfismo sexual com relação ao tamanho, sendo os machos maiores que as fêmeas (Johnson 2003, Masunari \& Swiech-Ayoub 2003, Colpo \& Negreiros-Fransozo 2004, Masunari \& Dissenha 2005, Castiglioni \& NeGreiros-Fransozo 2004a, b, 2006a, b). Para Johnson (2003), as fêmeas de caranguejos $U c a$ atingem tamanhos menores que os machos porque estas concentram suas energias para o desenvolvimento gonadal. Já os machos são geralmente maiores provavelmente porque, assim, têm mais chances ter sucesso na corte das fêmeas e de vencer combates intraespecíficos (CHRISTY \& Salmon 1984). Contudo, Costa \& Negreiros-Fransozo (2003) encontraram para a população de $U$. thayeri de Ubatuba fêmeas maiores do que os machos, como ocorreu no presente estudo.

Os animais do Manguezal de Itacuruçá apresentam tamanhos menores do que aqueles coletados em outros manguezais do Brasil. A exceção foi para $U$. leptodactyla e $U$. mordax que atingiram tamanhos maiores do que os coletados por Masunari \& Swiech-Ayoub (2003) em Itapoá, Santa Catarina e Masunari \& Dissenha (2005) em Guaratuba, Paraná. A população de $U$. thayeri também apresentou machos maiores que aqueles coletados por Costa \& Negreiros-Fransozo (2003) e Negreiros-Fransozo et al. (2003) e fêmeas maiores do que as analisadas por Negreiros-Fransozo et al. (2003), ambos em Ubatuba, São Paulo (Tab. III).

As variações no tamanho dos caranguejos de diferentes manguezais estão normalmente relacionados à plasticidade fenotípica, tipicamente influenciada por fatores ambientais como fotoperíodo, temperatura, precipitação, recursos alimentares e outros (Campbell \& Eagles 1983). A diferença na taxa de 

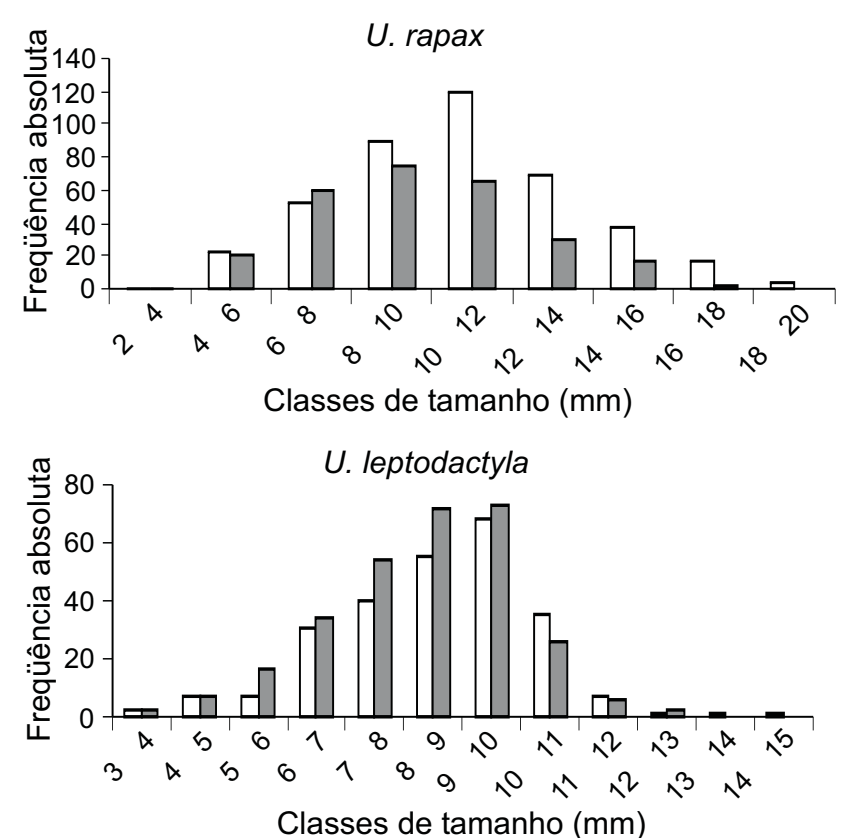

U. mordax
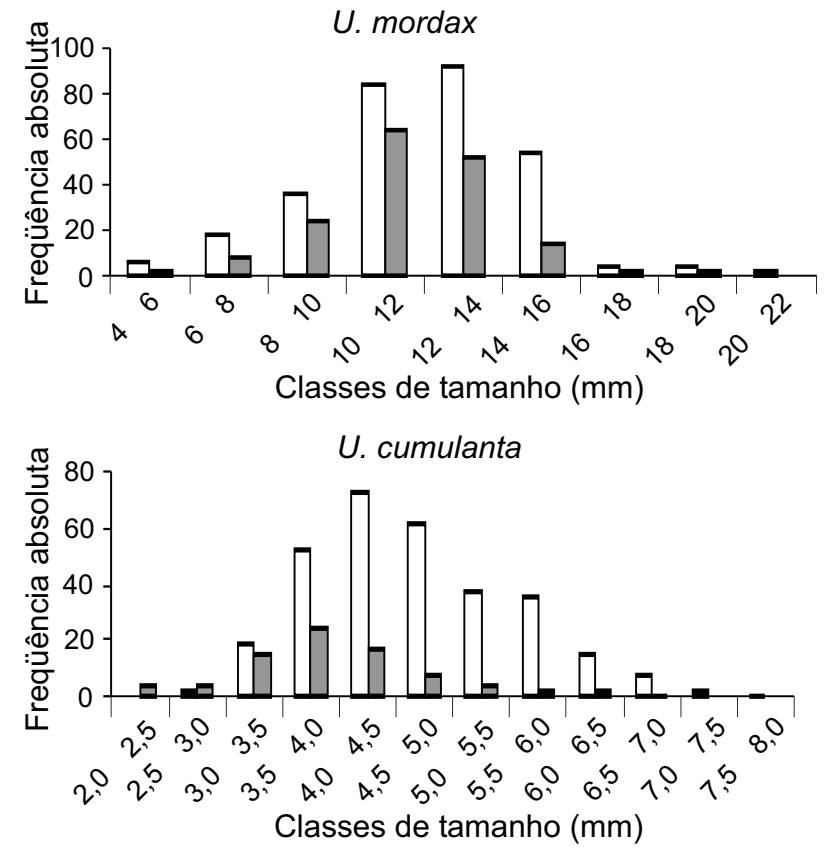

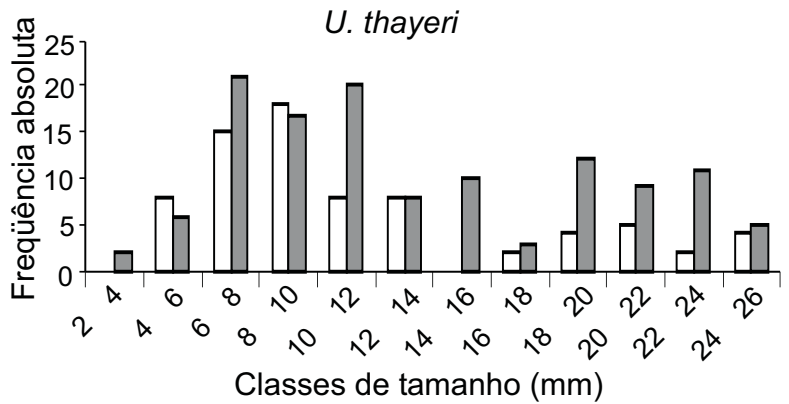

U. victoriana

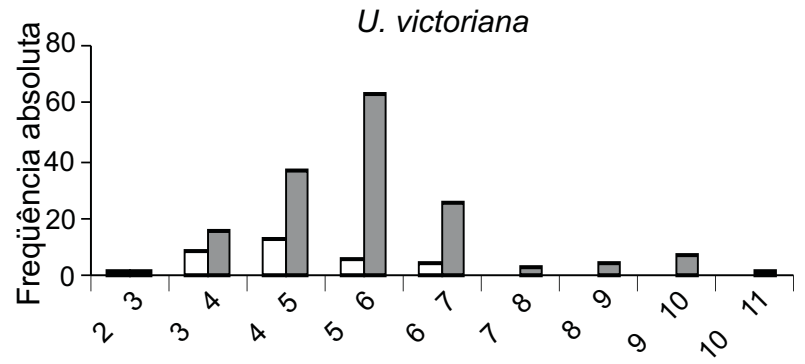

Classes de tamanho $(\mathrm{mm})$

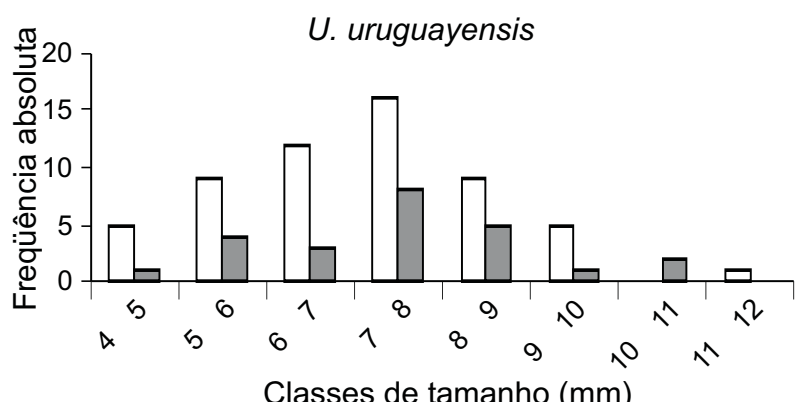

U. vocator

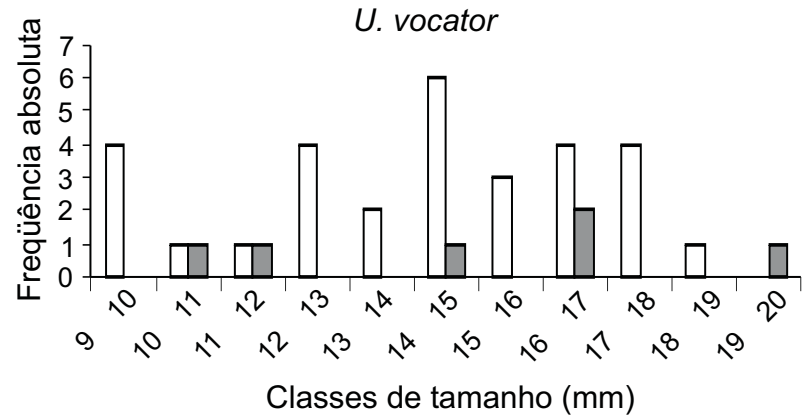

Figura 1. Distribuição de freqüência de tamanho dos exemplares do gênero Uca, coletados no Manguezal de Itacuruçá, no período de junho de 2005 a maio de 2006. Barras brancas = machos e Barras pretas = fêmeas.

crescimento pode estar relacionada à disponibilidade de alimento, mudanças no substrato e na densidade populacional (Hines 1989). Para Wenner et al. (1974), a disponibilidade de alimento pode promover diferenças no tamanho máximo que a população pode alcançar, porque quanto maior a disponibilidade deste recurso, maior é o crescimento do caranguejo. Esse fato pode ser confirmado por Colpo \& Negreiros-Fransozo (2004), que encontraram diferenças no tamanho das fêmeas ovígeras de Itamambuca, Indaiá e Itapanhaú, devido aos diferentes recursos alimentares entre as localidades.

Essa variação no tamanho dentro das espécies de $U c a$, pode estar relacionada, também, ao fato de que as populações 
Tabela III. Quadro comparativo da maior largura da carapaça de machos e fêmeas de espécies de Uca, capturados em estudos anteriormente realizados no Brasil.

\begin{tabular}{|c|c|c|c|c|}
\hline \multirow{2}{*}{ Espécies } & \multirow{2}{*}{ Localidade } & \multicolumn{2}{|c|}{ Maior largura da carapaça } & \multirow{2}{*}{ Autores } \\
\hline & & Machos & Fêmeas & \\
\hline \multirow[t]{2}{*}{ U. leptodactyla } & Itapoá, Santa Catarina & 11,5 & 10,70 & Masunari \& SWIECH-Ayoub (2003) \\
\hline & Itacuruçá, Rio de Janeiro & 14,3 & 12,60 & Presente estudo \\
\hline \multirow[t]{2}{*}{ U. mordax } & Guaratuba, Paraná & 20,0 & 18,90 & MASUNARI \& DISSENHA (2005) \\
\hline & Itacuruçá, Rio de Janeiro & 20,8 & 19,90 & Presente estudo \\
\hline \multirow[t]{6}{*}{ U. rapax } & Itamambuca, São Paulo & 28,3 & 27,30 & Castiglioni \& NeGreiros-Fransozo (2004a) \\
\hline & Ubatumirim, São Paulo & 24,2 & 22,00 & \\
\hline & Itamambuca, São Paulo & 26,6 & 25,50 & Castiglioni \& NeGreiros-Fransozo (2004b) \\
\hline & Ubatumirim, São Paulo & 24,9 & 22,50 & \\
\hline & Paraty, Rio de Janeiro & 22,9 & 21,20 & Castiglioni \& NeGreiros-Fransozo (2006a) \\
\hline & Itacuruçá, Rio de Janeiro & 19,5 & 16,80 & Presente estudo \\
\hline \multirow[t]{3}{*}{ U. thayeri } & Ubatuba, São Paulo & 25,2 & 28,40 & Costa \& NeGreiros-Fransozo (2003) \\
\hline & Ubatuba, São Paulo & 25,6 & 25,50 & NeGreiros-Fransozo et al. (2003) \\
\hline & Itacuruçá, Rio de Janeiro & 25,9 & 25,80 & Presente estudo \\
\hline \multirow[t]{2}{*}{ U. victoriana } & Vitória, Espírito Santo & 19,1 & 15,10 & VON HAGEN (1987) \\
\hline & Itacuruçá, Rio de Janeiro & 6,7 & 10,14 & Presente estudo \\
\hline \multirow[t]{4}{*}{ U. vocator } & Itapanhaú, São Paulo & 21,2 & 21,10 & Colpo \& NeGreiros-Fransozo (2004) \\
\hline & Indaiá, São Paulo & 23,7 & 23,70 & \\
\hline & Itamambuca, São Paulo & 27,0 & 24,80 & \\
\hline & Itacuruçá, Rio de Janeiro & 18,1 & 20,00 & Presente estudo \\
\hline
\end{tabular}

que ocorrem em regiões de baixa latitude (Região Tropical), atingem tamanhos maiores do que aquelas ocorrentes em latitudes acima de $24^{\circ}$ (Região Subtropical) (Masunari \& Dissenha 2005).

Para o Manguezal de Itacuruçá, provavelmente, dentre todos os fatores sugeridos, pode-se inferir também a especulação imobiliária, com construção de condomínios, que têm destruído parte do manguezal. Esta atividade tem como conseqüência a redução cada vez maior do manguezal, limitando a área de ecossistema ocupado por esses animais e redistribuindo a disponibilidade alimentar dos caranguejos.

A razão sexual em caranguejos Uca é freqüentemente diferente de 1:1, sendo os machos normalmente mais abundantes do que as fêmeas (GENONI 1985). Esse fato também foi verificado no presente estudo, concordando com o observado por Castiglioni \& Negreiros-Fransozo (2006a), para U. rapax, Masunari $\&$ Swiech-Ayoub (2003) para U. leptodactyla, Masunari \& Dissenha (2005) para U. mordax, Costa \& Negreiros-Fransozo (2003) para U. thayeri.

Para Valiela et al. (1974) e Emmerson (1994), os machos são mais fáceis de ser encontrados pelo fato de passar mais tempo na superfície do que as fêmeas, onde exibem comportamentos defensivos, de acasalamento e de alimentação por longos períodos, para compensar o fato de terem somente uma quela mais adaptada para apanhar o alimento.
A maior ocorrência de fêmeas pode ser associado a dois fatores: maior taxa de mortalidade de machos ou menor dificuldade na captura de fêmeas, devido a fatores comportamentais. Adicionalmente, a maior ocorrência de machos pode ser atribuído também ao comportamento das fêmeas na época da reprodução, onde ficam menos expostas ao ambiente externo (Souza \& FonToura 1993).

Thurman (1985) e Diaz \& Conde (1989) afirmam que razões sexuais desbalanceadas podem regular o tamanho da população, já que afetam seu potencial reprodutivo, sendo que este aumento ocorre quando há predominância de fêmeas na população (Christy \& SALMON 1984).

As populações consideradas homeostáticas, fisiologicamente e comportamentalmente, e que ocupam ambientes relativamente constantes, podem aclimatar-se a seu microambiente. Nesses casos, a razão sexual será aproximadamente equitativa ou levemente desviada a favor dos machos. Por outro lado, aquelas que se adaptam ou mudam geneticamente em resposta à variação ambiental, irão apresentar desvios a favor das fêmeas, como uma maneira de maximizar seu potencial evolutivo que é facilitado por uma distribuição desigual da intensidade de seleção entre os sexos (GEISEL 1972).

No presente estudo, verificou-se que a razão sexual das espécies analisadas apresentou semelhanças com estudos já realizados. Entretanto, os fatores comportamentais de repro- 
dução e alimentação parecem ser os mais adequados para explicar os desvios encontrados. A grande ocorrência de unimodalidade sugere que as populações, no presente estudo, se apresentam em equilíbrio, com taxas de recrutamento contínuo e de mortalidade constante como foi verificado por outros autores (Diaz \& Conde 1989, Hartnoll \& Bryant 1990). Esse padrão de distribuição é bastante comum em populações de decápodes de regiões tropicais, onde não ocorrem variações climáticas acentuadas (WARner 1967, Diaz \& CONDE 1989).

As distribuições sem um padrão definido encontrado para as populações de $U$. thayeri e $U$. vocator podem ser explicadas pelo fato de que $U$. thayeri encontrava-se numa das regiões mais afetadas pelo avanço imobiliário. Mudanças no substrato foram observadas e isso pode estar induzindo os animais a migrar para outras regiões mais propícias. É possível, todavia, que o pequeno número de $U$. vocator coletados não possibilitou verificar a real distribuição da população.

De maneira geral, as classes de maior tamanho tiveram uma predominância de machos, fato também verificado por Abele et al. (1986) para o caranguejo grapsídeo Pachygrapsus transversus (Gibbes, 1850). Conforme WARner (1967), Wenner (1972), Thurman II (1985) e Diaz \& Conde (1989), a predominância de machos nas classes de maior tamanho pode estar relacionada ao crescimento mais lento das fêmeas, após a puberdade, em relação aos machos.

Pode-se concluir que o Manguezal de Itacuruçá apresenta as condições necessárias para o desenvolvimento das diferentes espécies de Uca. Contudo, o fato da maioria das espécies apresentarem tamanhos menores do que em outros manguezais do Brasil, infere que fatores antropogênicos vêm reduzindo o habitat e escasseando os recursos alimentares dessas espécies, influenciando sobre essas populações.

Portanto, há necessidade de estudos para o manejo e conservação dessas espécies no Manguezal de Itacuruçá, uma vez que são raros os registros na literatura, da co-existência dessas várias espécies de Uca num mesmo manguezal.

\section{LITERATURA CITADA}

Abele, L.G.; P.J. Campanella \& M. Salmon. 1986. Natural history and social organization of the semiterrestrial grapsidae crab Pachygrapsus transversus (Gibbes). Journal of Experimental Marine Biology and Ecology 104: 153-170.

Allen, A.A. \& H.A. Curran. 1974. Biogenic sedimentary structures produced by crabs in lagoon margin and salt marsh enviromnents near Beautfort, North Carolina. Journal of Sedimentary Petrology 44: 538-548.

Ashton, E.C.; D.J. Macintoch \& P.J. Hoghart. 2003. A baseline study of the diversity and community ecology of crab and molluscan macrofauna in the Sematan mangrove forest. Sarawak, Malaysia. Journal of Tropical Ecology 19: 127142.

BedÊ, L.M.; L.M.Y. Oshiro \& G.A.S. Melo. 2007. Observation on the occurrence of Uca victoriana von Hagen (Decapoda,
Brachyura, Ocypodidae) on the Coast of Rio de Janeiro, Brazil. Brazilian Journal of Biology 67 (4): 799-800.

Botto, F. \& O. Iribarne. 2000. Constranting effects of two burrowing crabs (Chasmagnathus granulata and Uca uruguayensis) on sediment composition and transport in estuarine environments. Estuarine Coastal Marine Science 51: 141-151.

Campbell, A. \& M.D. Eagles. 1983. Size maturity and fecundity of rock crabs, Cancer irroratus, from the Bay of Fundy and southwestern Nova Scotia. Fishery Bulletin 81 (2): 357-362.

Castiglioni, D.S. \& M.L. Negreiros-Fransozo. 2004a. Comparative analysis of the relative growth of Uca rapax (Smith) (Crustacea, Ocypodidae) from two mangroves in São Paulo, Brazil. Revista Brasileira de Zoologia 21 (1): 137-144.

Castiglioni, D.S. \& M.L. Negreiros-Fransozo. 2004b. Somatic growth of the mudflat fiddler crab Uca rapax (Smith, 1870) (Brachyura: Ocypodidae) from two tropical mangroves in Brazil. Universidade y Ciencia 20 (39): 15-22.

Castiglioni, D.S. \& M.L. Negreiros-Fransozo. 2006a. Ciclo reprodutivo do caranguejo violinista Uca rapax (Smith) (Crustacea, Brachyura, Ocypodidae) habitante de um estuário degradado em Paraty, Rio de Janeiro, Brasil. Revista Brasileira de Zoologia 23 (2): 331-339.

Castiglioni, D.S. \& M.L. Negreiros-Fransozo. 2006b. Physiologic sexual maturity of the fiddler crab Uca rapax (Smith, 1870) (Crustacea, Ocypodidae) from two mangroves in Ubatuba, Brazil. Brazilian Archives of Biology and Technology 49 (2): 239-248.

Christy, J. H. \& SAlmon, M. 1984. Ecology and evolution of mating systems of fiddler crabs (genus Uca). Biological Reviews 59: 483-599.

Colpo, K.D. \& M.L. Negreiros-Fransozo. 2004. Comparasion of the population structure of the fiddler crab Uca vocator (Herbst, 1804) from three subtropical mangrove forests. Scientia Marina 68 (1): 139-146.

Costa, T. M.. \& M.L. Negreiros-Fransozo. 2003. Population biology of Uca thayeri Rathbun, 1900 (Brachyura, Ocypodidae) in a subtropical south American mangrove area: results from transect and catch-per-unit-techniques. Crustaceana 75 (10) 1201-1218.

Costa, T.M.; A.A.J. Silva \& M.L. Negreiros-Fransozo. 2006. Reproductive pattern comparison of $U$. thayeri Rathbun, 1900 and U. uruguayensis Nobili, 1901 (Crustacea, Decapoda, Ocypodidae). Brazilian Archives and Technology 49 (1): 117-123.

Crane, J. 1975. Fiddler crabs of the world. Ocypodidae: genus Uca. Princeton, Priceton University Press, XXIV+736p.

Diaz, H. \& J.E. Conde. 1989. Population dynamics and life history of the mangrove crab Aratus pisonii (Brachura, Grapsidae) in a marine environment. Bulletin of Marine Science 45: 149-163.

EMmERSon, W.D. 1994. Seasonal breeding cycles and sex ratio of eight species of crabs from Magazana, a mangrove estuary 
in Transkei, southern Africa. Journal of Crustacean Biology 14 (3): 568-578.

Frith, D.W. \& S. Brunenmeister. 1980. Ecological and population studies od fiddler crabs (Ocypodidae, genus Uca) on a mangrove shore at Phuket Island, Western Peninsular Thailand. Crustaceana 39 (2): 157-183.

Geisel, J.T. 1972. Sex ratio, rate of evolution, and environmental heterogeneity. American Naturalist 106: 380-387.

Genoni, G.P. 1985. Food limitation in salt marsh fiddler crabs Uca rapax (Smith) (Decapoda, Ocypodidae). Journal of Experimental Marine Biology and Ecology 87: 97-110.

HartNoll, R.G. \& A.D. BRyANT. 1990. Size-frequency distribuitions in decapod Crustacea - The quick, the dead, and the castoffs. Journal of Crustacean Biology 10 (1): 14-19.

Hines, A.H. 1989. Geografic variation in size at maturity in brachyuran crabs. Bulletin of Marine Science 45 (2): 356-368.

Johnson, P.T.J. 2003. Biased sex ratios in fiddler crabs (Brachyura, Ocypodidae): A review and evaluation of the influence of sampling method, size class, and sex-specific mortality. Crustaceana 76: 559-580.

Jones, M.B. \& M.J. Simons. 1983. Latudinal variation in repdroductive characteristics of a mud crab Helice grassa (Grapsidae). Bulletin of Marine Science 33 (3): 656-670.

LEE, S.Y. 1999. The effect of mangrove leaf litter enrichment on macrobenthic colonization of defauned sand substrates. Estuarine Coastal Marine Science 49: 703-712.

Masunari, S. \& N. Dissenha. 2005. Alometria no crescimento de Uca mordax (Smith) (Crustacea, Decapoda, Ocypodidae) na Baía de Guaratuba, Paraná, Brasil. Revista Brasileira de Zoologia 22 (4): 984-990.

Masunari, S. \& B.P. Swiech-Ayoub. 2003. Crescimento relativo em Uca leptodactyla Rathbun (Crustacea, Decapoda, Ocypodidae). Revista Brasileira de Zoologia 20 (3): 487-491.

Melo, G.A.S. 1996. Manual de identificação dos Brachyura (caranguejos e siris) do litoral brasileiro. São Paulo, Ed. Plêiade Fapesp, 603p.

Meziane, T.; M.C. Sanabe \& M. Tsuchiya. 2002. Role of fiddler crabs of a subtropical intertidal flat on the fate of sedimen- tary fatty acids. Journal of Experimental Marine Biology and Ecology 270: 191-201.

Negreiros-Fransozo, M.L.; K.D. Colpo \& T.M. Costa. 2003. Allometric growth in the fiddler crab Uca thayeri (Brachyura, Ocypodidae) from a subtropical mangrove. Journal of Crustacean Biology 23 (2): 273-279.

Oshiro, L.M.Y.; R. SiLva \& Z.S. Silva. 1998. Composição da fauna de braquiúros (Crustacea Decapoda) nos manguezais da Baía de Sepetiba-RJ. Nauplius 6: 31-40.

Santos, S.; M.L. Negreiros-Fransozo \& A. Fransozo. 1995. Morphometric relationships and maturation in Portunus spinimanus Latreille, 1819 (Crustacea, Brachyura, Portunidae). Revista Brasileira de Biologia 55 (4): 545-553.

SouzA, G.D. \& N.F. Fontoura. 1993. Estrutura populacional e fecundidade de Pachygrapsus transversus (Saussure, 1858), no molhe do rio Tramandaí, Rio Grande do Sul, Brasil (Crustacea, Decapoda, Grapsidae). Comunicações do Museu de Ciências e Tecnologia 52: 29-37.

Skov, M.W.; J.P. Vannini; R.G. Shunnula; R.G. Hartnoll \& S. CANNICH. 2002. Quantifying the density of mangrove crabs: Ocypodidae and Grapsidae. Marine Biology 141: 725-732.

Thurman II, C.L. 1985. Reproductive biology and population structure of the crab Uca subcilindrica (Stimpson). Biological Bulletin 166: 215-229.

Valiela, I.; D.F. Babiec; W. Atherton; S. Seitzinger \& C. Kebs. 1974. Some consequences of sexual dimorphism: feeding in male and female fiddler crabs, Uca pugnax (Smith). Biological Bulletin 147: 652-660.

WARnER, G.F. 1967. The life history of mangrove tree crab, Aratus pisoni. Journal of Zoology 153: 321-335.

Wenner, A.M.; H.M. Page \& P.R. Siegel. 1972. Variation in size ot onset of egg production, p. 149-163. In: A.M. WENNER (Ed.). Factors in adult growth. Rotterdam, A.A. Balkema, 362p.

Wenner, A.M.; C. Fusaro \& A. Oaten. 1974. Size at onset of sexual maturity and growth rate in crustacean populations. Canadian Journal of Zoology 52 (9): 1095-1106.

ZAR, J.H. 1984. Biostatiscal Analysis. England, Prentice-Hall, $2^{\text {nd }}$ ed., $719 \mathrm{p}$.

Submitted: 13.XII.2007; Accepted: 29.XI.2008.

Editorial responsibility: Maria Lúcia Negreiros Fransozo

Revista Brasileira de Zoologia 25 (4): 601-607, December, 2008 